\title{
Effect of roll number on the statistics of turbulent Taylor-Couette flow
}

\author{
Rodolfo Ostilla-Mónico, ${ }^{1,2,{ }^{*}}$ Detlef Lohse, ${ }^{1}$ and Roberto Verzicco ${ }^{3,1}$ \\ ${ }^{1}$ Physics of Fluids Group, Faculty of Science and Technology, MESA+ Research Institute, and J. M. Burgers \\ Centre for Fluid Dynamics, University of Twente, PO Box 217, 7500 AE Enschede, The Netherlands \\ ${ }^{2}$ School of Engineering and Applied Sciences, Harvard University, Cambridge, Massachusetts, USA \\ ${ }^{3}$ Dipartimento di Ingegneria Industriale, University of Rome "Tor Vergata," Via del Politecnico 1, \\ Rome 00133, Italy
}

(Received 17 February 2016; published 7 September 2016)

\begin{abstract}
A series of direct numerical simulations in large computational domains has been performed in order to probe the spatial feature robustness of the Taylor rolls in turbulent Taylor-Couette flow. The latter is the flow between two coaxial independently rotating cylinders of radius $r_{i}$ and $r_{o}$, respectively. Large axial aspect ratios $\Gamma=7-8$ [with $\Gamma=L /\left(r_{o}-r_{i}\right)$, and $L$ the axial length of the domain] and a simulation with $\Gamma=14$ were used in order to allow the system to select the most unstable wave number and to possibly develop multiple states. The radius ratio was taken as $\eta=r_{i} / r_{o}=0.909$, the inner cylinder Reynolds number was fixed to $\operatorname{Re}_{i}=3.4 \times 10^{4}$, and the outer cylinder was kept stationary, resulting in a frictional Reynolds number of $\operatorname{Re}_{\tau} \approx 500$, except for the $\Gamma=14$ simulation where $\operatorname{Re}_{i}=1.5 \times 10^{4}$ and $\operatorname{Re}_{\tau} \approx 240$. The large-scale rolls were found to remain axially pinned for all simulations. Depending on the initial conditions, stable solutions with different number of rolls $n_{r}$ and roll wavelength $\lambda_{z}$ were found for $\Gamma=7$. The effect of $\lambda_{z}$ and $n_{r}$ on the statistics was quantified. The torque and mean flow statistics were found to be independent of both $\lambda_{z}$ and $n_{r}$, while the velocity fluctuations and energy spectra showed some box-size dependence. Finally, the axial velocity spectra were found to have a very sharp dropoff for wavelengths larger than $\lambda_{z}$, while for the small wavelengths they collapse.
\end{abstract}

DOI: 10.1103/PhysRevFluids.1.054402

\section{INTRODUCTION}

Taylor-Couette (TC) flow, the flow between two independently rotating coaxial cylinders, is one of the paradigmatical systems in fluid mechanics, due to both its high simplicity and its applications in technology and nature. While the low Reynolds number regime of TC flow has been studied in great detail for decades [1], the large Reynolds number regime remained relatively unexplored until the last few years [2]. Only recently has enough computational power become available that fully resolved simulations reaching the so-called "ultimate" regime of TC flow, where both boundary layers and bulk are turbulent, have become a possibility [3].

In the absence of viscosity, TC flow is linearly unstable if $\left|r_{i}^{2} \omega_{i}\right|>\left|r_{o}^{2} \omega_{o}\right|$, where $r_{i}$ and $r_{o}$ are the inner and outer cylinder radia, respectively, and $\omega_{i}$ and $\omega_{o}$ are the inner and outer cylinder angular velocities, respectively. Due to this instability, a series of transitions take place when the inner cylinder is rotated with increasing speed. For very small driving, the flow is purely azimuthal. Once the driving is large enough to overcome the viscous damping, this purely azimuthal flow becomes centrifugally unstable and stationary large-scale structures fill the entire gap, effectively redistributing angular momentum. These structures are called Taylor rolls after the seminal work by Taylor [4]. Further increasing the driving causes the onset of time dependence, and the Taylor rolls to transition to wavy Taylor rolls first, then to modulated Taylor rolls, and finally to turbulent Taylor rolls [5]. At the highest Reynolds numbers achieved in both simulations, i.e., $\operatorname{Re} \sim O\left(10^{5}\right)$, and experiments,

*rostillamonico@g.harvard.edu 
i.e., $\operatorname{Re} \sim O\left(10^{6}\right)$, an axially stationary signature of these rolls can be observed [6,7]. This signature is present only in certain regions of the high Reynolds number TC flow parameter space, mainly depending on the radius ratio of the system $\eta=r_{i} / r_{o}$ and the rotation ratio $\mu=\omega_{o} / \omega_{i}$ [8].

Experimental realizations of TC flow necessarily have end caps at the top and bottom of the systems, which may be fixing the position of the rolls in the axial direction. The first photographs of experimentally pinned Taylor rolls were provided by Coles [9], at Reynolds numbers of $\operatorname{Re} \sim O\left(10^{4}\right)$. Further studies by Benjamin and Mullin [10], Andereck et al. [5], Lathrop et al. [11], Martinez-Arias et al. [12], and Huisman et al. [6] have repeatedly shown, in several experiments up to $\operatorname{Re} \sim O\left(10^{6}\right)$, that the rolls are pinned, and they have also shown the multiplicity of roll states and the crucial role of the initial conditions and hysteresis in determining the aspect ratio of the rolls. A detailed study of roll-size hysteresis at $\operatorname{Re} \sim O\left(10^{6}\right)$ was performed by van der Veen et al. [13]. Simulations use periodic boundary conditions, which a priori should not fix the position of these rolls. The puzzling axial pinning of the Taylor rolls, observed in Refs. $[7,8]$ and the resulting lack of statistical axial homogeniety of the axially periodic direct numerical simulations (DNSs), was speculated to be caused by an insufficient axial extent of the domain [14]. "Small" computational boxes have been used in TC flow to be able to perform the high Reynolds number simulations by both Brauckmann et al. [15-17] and Ostilla-Mónico et al. [3,7,8]. In these simulations, the aspect ratio $\Gamma=L_{z} /\left(r_{o}-r_{i}\right)$, where $L_{z}$ is the axial periodicity length, was limited to $\Gamma \approx 2$, enough to fit a single-roll pair. In addition, a rotational symmetry of order $n_{\text {sym }}$ was imposed to reduce the azimuthal extent of the domain at the midgap $L_{x}$ to $L_{x} / d \approx \pi$. To assess the validity of these computational boxes, a systematic study was conducted by Ostilla-Mónico et al. [18], who found that these small boxes were sufficient to produce box-independent statistics for the torque and mean velocity profiles for pure inner cylinder rotation at $\operatorname{Re}_{i}=10^{5}$, where $\mathrm{Re}_{i}$ is the inner cylinder Reynolds number defined as $\operatorname{Re}_{i}=\left(r_{o}-r_{i}\right) \omega_{i} r_{i} / v$, and $v$ is the kinematic viscosity of the fluid. However, the velocity fluctuations were found to be box-dependent, even for the largest boxes considered. This trend was in agreement to what was observed in DNSs of channel flow, i.e., the pressure-driven flow between two parallel plates, by Lozano-Durán and Jiménez [19].

A second raised issue was that in Ref. [18] only cases with a single-roll pair were considered. The aspect ratio $\Gamma$ was varied between 2 and 4, and in the simulations, a single, axially stationary roll pair was observed, whose wavelength $\lambda_{z}$ was found to grow with increasing $\Gamma$ up to $\lambda_{z}=4$. For even larger $\Gamma$, one could expect more than a single-roll pair to form and even to have multiple "states," i.e., different number of turbulent roll pairs depending on the initial conditions, which could affect the statistics dramatically [6].

\section{NUMERICAL DETAILS}

In this paper we conduct a series of DNSs of TC flow using the incompressible Navier-Stokes equations in computational boxes with very large axial and azimuthal extents to answer the issues previously raised. These boxes can fit more than a turbulent Taylor roll pair and thus can be used to assess the effect of the roll number on high-order statistics. These simulations were performed using an energy-conserving second-order finite difference code [20,21], with fractional time stepping. The radius ratio was fixed to $\eta=0.909$, in the parameter space region where small boxes showed Taylor rolls to be strong and axially pinned. A rotational symmetry $n_{\text {sym }}=5$ was imposed, meaning that only a fifth of the cylinder was simulated, and periodic boundary conditions were used in the azimuthal direction. This is a smaller $n_{\text {sym }}$ than previously used for this $\eta\left(n_{\text {sym }}=20\right)$, so the domain was four times larger in the azimuthal direction. In the axial direction, $\Gamma$ was chosen to be either $\Gamma=7$ or $\Gamma=8$. This results in a streamwise extent of the box at midgap of $8.4 \pi$ half-gaps and an axial extent of the box of $14(4.5 \pi)$ or $16(5.1 \pi)$ half-gaps, comparable to the large boxes run in plane Couette (PC) flow simulations [22,23]. PC flow is the flow between two parallel and independently moving plates. Rotating PC flow, where the two plates can also rotate about an axis parallel to them, is the limiting case of TC flow when $\eta \rightarrow 1$. Rotating PC flow has two control parameters, the shear Reynolds number and the rotation or Rossby number, which are equivalent to the shear Reynolds 
TABLE I. Details of the numerical simulations. The first column is the case name, the second column is the aspect ratio $\Gamma$, and the third column is the roll wavelength $\lambda_{z}=\Gamma / n_{r}$, where $n_{r} \in \mathbb{N}$ is the number of rolls. The fourth column is the imposed rotational symmetry. The fifth to seventh columns represent the amount of points in the azimuthal, radial, and axial directions. The eighth column represents the nondimensional torque $\mathrm{Nu}_{\omega}$. The ninth column is the frictional Reynolds number at the inner cylinder. The second to last column shows the averaging time for statistics. The last column refers to the line colors in Figs. 5 to 8 .

\begin{tabular}{lcccccccccl}
\hline \hline Case & $\Gamma$ & $\lambda_{z}$ & $n_{\text {sym }}$ & $N_{\theta}$ & $N_{r}$ & $N_{z}$ & $\mathrm{Nu}_{\omega}$ & $\mathrm{Re}_{\tau, i}$ & $t_{\text {stat }} U_{i} / d$ & Color of lines \\
\hline G2R1 & 2.33 & 2.33 & 20 & 384 & 512 & 768 & 24.3 & 490 & 233 & Blue solid \\
G7R3 & 7 & 2.33 & 5 & 1536 & 512 & 2560 & 24.3 & 490 & 78 & Orangish solid \\
G7R2 & 7 & 3.5 & 5 & 1536 & 512 & 2976 & 26.3 & 510 & 121 & Ocre dashed \\
G8R3 & 8 & 2.66 & 5 & 1536 & 512 & 2976 & 24.9 & 500 & 104 & Purple dash-dot \\
G14R7 & 14 & 2 & 2 & 1536 & 256 & 2560 & 13.5 & 240 & 487 & None \\
\hline \hline
\end{tabular}

number and Rossby number for TC flow as defined in Ref. [24]. Unlike TC flow, PC flow does require large computational boxes because the decorrelation lengths are much longer [25].

In these simulations, the inner cylinder Reynolds number was set to $\operatorname{Re}_{i}=3.4 \times 10^{4}$, while the outer cylinder was kept stationary. This resulted in an inner cylinder frictional Reynolds number $\operatorname{Re}_{\tau}=u_{\tau, i}\left(r_{o}-r_{i}\right) /(2 v) \approx 500$, where $u_{\tau, i}$ is the inner frictional velocity $u_{\tau, i}=\left(\tau_{i} / \rho\right)^{1 / 2}$, $\tau_{i}$ the shear stress at the inner cylinder, and $\rho$ the fluid density. The outer cylinder frictional velocity (Reynolds number) is given by $u_{\tau, o}=\eta u_{\tau, i}\left(\operatorname{Re}_{\tau, o}=\eta \operatorname{Re}_{\tau, i}\right)$. For convenience, we also define the nondimensional distance from the wall $\tilde{r}=\left(r-r_{i}\right) /\left(r_{o}-r_{i}\right)$, the nondimensional axial coordinate $\tilde{z}=z /\left(r_{o}-r_{i}\right)$, and the nondimensional azimuthal coordinate at the midgap $\tilde{x}=\frac{1}{2}\left(r_{i}+r_{o}\right) \theta /\left(r_{o}-r_{i}\right)$.

The simulations were run in a rotating frame of reference similar to the one proposed by Dubrulle et al. [24], such that the velocity at both cylinders was equal to half of the characteristic velocity, and of opposite sign, and thus the mean velocity was equal to zero at the midgap to reduce as far as possible the dispersion errors in the spectra due to the use of finite differences and allows for larger time steps for the same $\Delta t^{+}$[26]. We note that the Reynolds numbers simulated here are a factor three smaller than the one considered for the previous box-size comparison in Ref. [18], i.e., $\operatorname{Re}_{\tau} \approx 1400$ and $\operatorname{Re}_{i}=10^{5}$, but this is necessary to keep the computational costs manageable with large computational domains. With this Reynolds number, the largest grid resolution used was $N_{\theta} \times N_{r} \times N_{z}=1536 \times 512 \times 2926$ for the $\Gamma=8$ box, i.e., over two billion points. Full details of the numerical resolutions used are in Table I. Points were clustered in the radial direction using a clipped Chebychev distribution and homogeneously distributed in the other two directions, which resulted in a resolution in inner cylinder wall units of $r_{i} \Delta \theta^{+}=8.6, \Delta r^{+} \in(0.4,2.9)$ and $\Delta z^{+}=2.7$, where wall units are defined using $u_{\tau}$ and $\delta_{v}=v / u_{\tau}$. The time step of the simulations was taken so that $\Delta t^{+}=0.4$, and the simulations were ran for over 100 large eddy turnover times based on $d /\left(r_{i} \omega_{i}\right)$, equivalent to at least six turnover times based on the frictional velocity and the half-gap, i.e., $d /\left(2 u_{\tau}\right)$. This resulted in a wall time of 3 weeks on 480 cores. The statistical convergence of the solution can be estimated by noting that the angular velocity current [27] $J^{\omega}=$ $r^{3}\left(\left\langle u_{r} \omega\right\rangle_{\theta, t, z}-v \partial_{r}\langle\omega\rangle_{\theta, t, z}\right)$ is radially constant to within $1 \%$, where the $\langle\ldots\rangle_{x_{i}}$ operator indicates averaging with respect to the independent variable $x_{i}$.

\section{RESULTS}

First, two simulations, one for $\Gamma=7$ and one for $\Gamma=8$, were started from initial conditions consisting of a quiescent fluid with some added white noise in the velocity fields. During a transient which lasted around $200 d /\left(r_{i} \omega_{i}\right)$ turnover times, large-scale axially stationary rolls were seen to form, two pairs in the case of $\Gamma=7$ (this would be the G7R2 simulation) and three pairs in the case of $\Gamma=8$ (G8R3), resulting in roll wavelengths of $\lambda_{z}=3.5$ and $\lambda_{z}=2.66$. Figure 1 shows a 


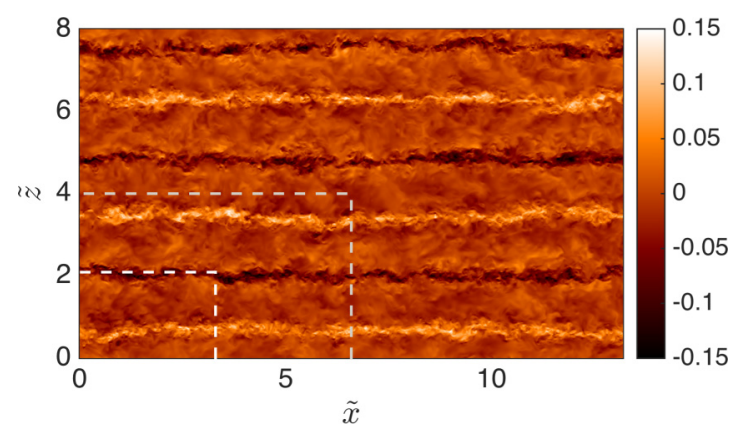

FIG. 1. Instantaneous angular velocity at the midgap for the $\Gamma=8$ simulation. $\tilde{z}=z / d$ is the axial direction and $\tilde{x}=r_{a} \theta d$ the rescaled azimuthal direction with $r_{a}$ the midgap radius. The signature of the Taylor rolls can be seen as a large, stripy pattern, which appears to be fixed in the axial position for the entire azimuthal extent. For comparison, the (smaller) white red line indicates the typical size of a small TC flow computational box of Ref. [7]. The (larger) light gray dashed line indicates the largest box simulated in Ref. [18].

pseudocolor plot of the azimuthal velocity at the midgap for the largest box simulated and a sketch of the "small" boxes normally used for TC flow DNS and of the largest box of Ref. [18] for comparison purposes.

After this, the statistically stationary flow field from the G8R3 case was rescaled to fit into a $\Gamma=7$ box and used as initial condition for a third case, G7R3. This case was advanced in time for around $300 \mathrm{~d} /\left(r_{i} \omega_{i}\right)$, around 200 turnover times to overcome the transient and 78 more to take statistics. The resulting statistically stationary flow field from this third simulation had a different roll state, i.e., three-roll pairs, with wavelength $\lambda_{z}=2.33$, different from the two-roll pair $\lambda_{z}=3.5$ case arising from the white noise initial conditions. We note that this axial rescaling, while convenient for generating the desired roll states, does not always work. This method cannot generate unphysical wavelengths since if the rolls are stretched or contracted beyond physical solutions, they will merge or break up. Finally, to asses the effect of the amount of rolls on the flow statistics, a fourth simulation (G2R1) with $\Gamma=2.33$ and a single-roll pair of $\lambda_{z}=2.33$ was performed to compare against the G7R3 case with three-roll pairs of $\lambda_{z}=2.33$.

To ensure that the rolls were indeed fixed even for larger computational boxes, one more case denoted by G14R7 was run for $\mathrm{Re}_{i}=1.57 \times 10^{4}$. The box parameters were $\Gamma=14$ and $n_{\text {sym }}=2$, resulting in a computational box of $21 \pi \times 2 \times 9 \pi$ half-gaps. The resulting inner cylinder frictional Reynolds number was $\operatorname{Re}_{\tau} \approx 240$. As $\operatorname{Re}_{\tau}$ was smaller, this allowed for coarser grids and larger time steps while still maintaining the accuracy of the simulations. The simulations were started from white noise initial conditions, and a state with seven rolls formed. This case was run for an even longer time to collect statistics: 487 time units based on the large-eddy turnover time or 29 time units based on the frictional velocity and the half-gap.

Table I provides a summary of the simulations ran, and the resulting frictional Reynolds numbers and nondimensional angular velocity current (torque) pseudo-Nusselt number $\mathrm{Nu}_{\omega}=J^{\omega} / J_{p a}^{\omega}$ [27], where $J_{p a}^{\omega}$ is the angular velocity current for the purely azimuthal case. A very weak variation of the frictional Reynolds number can be seen, which is consistent with the weak dependence of the torque on the roll wavelength for the Reynolds number considered here [8]. $\mathrm{The}^{\mathrm{Nu}} \omega$ for the singleand three-roll pair cases at the same $\lambda_{z}$ coincides within statistical convergence, consistent with the low Reynolds number result of Ref. [15]. As a consequence, the resulting $\operatorname{Re}_{\tau}$, which scales as $\operatorname{Re}_{\tau} \sim \sqrt{\operatorname{Re}_{i} \mathrm{Nu}_{\omega}}$, is also independent of the number of rolls. The small dependence of $\mathrm{Nu}_{\omega}$ on $\lambda_{z}$ vanishes for larger Reynolds number, so box-independent values for the torque can be obtained for $\Gamma=2[8,18]$.

Figure 2 shows a comparison of the azimuthally and temporally averaged azimuthal velocity for both $\Gamma=7$ cases. As noted previously, "eppur non si muove": even for these simulations 

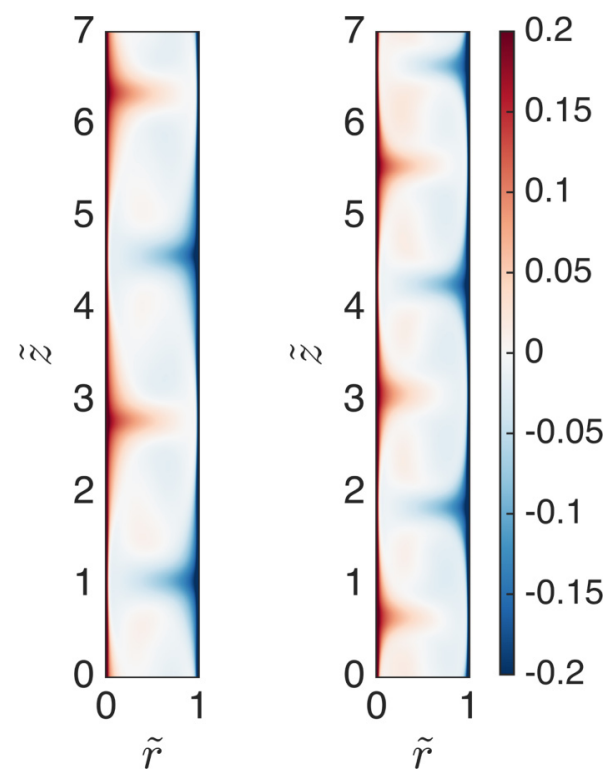

FIG. 2. Azimuthally and temporally averaged azimuthal velocity for the G7R2 and G7R3 cases, starting from a random velocity field (left, G7R2) or from a rescaled velocity field with three rolls (right, G7R3 case). From the characteristic signature of the rolls, the different roll wavelength can be appreciated. Both cases have been run for about 100 large eddy turnover times based on the large-eddy turnover time and appear to be stable.

with very large computational boxes, the large-scale rolls are still axially fixed. We note that the roll wavelengths which result from the white noise initial conditions in large $\Gamma$ simulations are those of "tall" rolls for the higher Reynolds number case and "square" for the lower Reynolds number case. Indeed, it seems to be the case that for increasing Reynolds numbers the characteristic roll wavelength, and thus the axial decorrelation length, increases. This effect was already noted previously in TC flow by several authors $[8,12]$ who observed "tall" rolls only for high Reynolds numbers and "wide" rolls only at low Reynolds numbers. Indeed, experimentally Huisman et al. [6] observed rolls with wavelengths up to $\lambda_{z}=3.9$ at $\operatorname{Re} \sim O\left(10^{6}\right)$ with $\Gamma=11.7$, sufficiently large not to substantially constrain the roll wavelength. Theoretical results show that the most unstable axial wave number is unbounded with increasing Reynolds number [28]. Furthermore, an increase of the axial decorrelation for the azimuthal velocity length with increasing $\operatorname{Re}_{\tau}$ in DNSs of plane Couette flow was seen in Refs. [22,23]. We can thus speculate that for infinite Reynolds number, the roll wavelength becomes infinitely large, and thus we recover the axial symmetry of the system in a statistical sense.

To further demonstrate the axial pinning of the rolls, in Fig. 3 we show the azimuthally and temporally averaged azimuthal velocity for the G17R7 case. Statistics for this case have been taken for a much longer time than the other four cases, and still the rolls, which have a square-aspect ratio here, appear to be fixed. The axial extent of this box $(\approx 9 \pi$ half-gaps $)$ is larger than the one

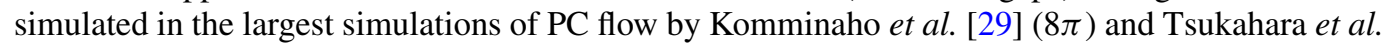

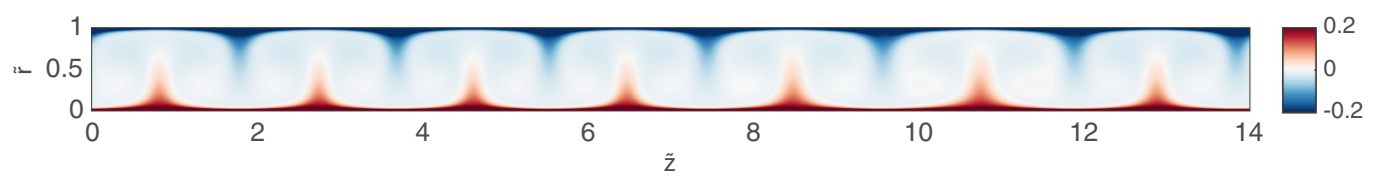

FIG. 3. Azimuthally and temporally averaged azimuthal velocity for the G14R7 case. 


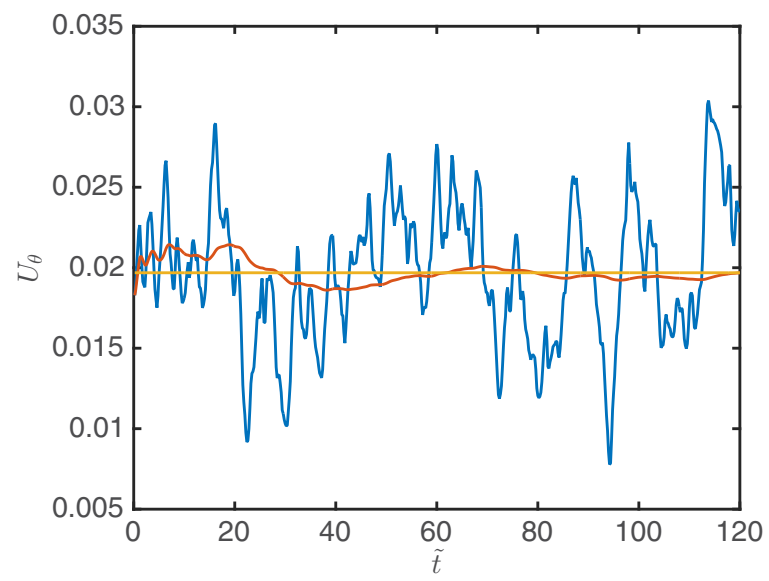

FIG. 4. The blue line shows the temporal evolution of the azimuthally averaged azimuthal velocity at a point in the midgap inside the ejection region of a roll. The orangish line shows the running average of this quantity, and the ochre horizontal line shows the final average. The data used are for only 120 time units, as these are representative of all simulations in this paper.

$[25](\approx 8.2 \pi)$, and we expect it to be sufficient to show that the large-scale structures are indeed pinned. To ensure that we are not missing a slow evolution of the rolls, Fig. 4 shows the azimuthally averaged velocity at a midgap point inside a roll, as well as the running mean and the final value of the mean. Small-amplitude temporal fluctuations can be seen, with a characteristic time scale of $\sim 30$ nondimensional time units. This coincides with the frictional time scale $O\left(u_{\tau} / d\right)$. However, these oscillations are too small to be an unpinning of the rolls, which would amount for much larger variations of velocity. In addition, we note that we have shown data only for 120 time units, instead of the 487 time units that statistics were taken for the G14R7 case, to ensure fair comparison to the other cases in this paper. The very large time average window of the G14R7 case has resulted in a spatial convergence of the angular velocity current, which is constant to less than $0.5 \%$, a more stringent requirement than other cases.

Figure 5 shows the average streamwise velocity profile at the inner cylinder in wall units, i.e., $u^{+}=\left(r_{i} \omega_{i}-\left\langle u_{\theta}\right\rangle_{\theta, z, t}\right) / u_{\tau, i}$ against $r^{+}=\left(r-r_{i}\right) / \delta_{v, i}$. We can see a clear dependence on $\lambda_{z}$ and on the roll wavelength, but again not on the number of rolls. This dependence on $\lambda_{z}$ becomes weaker for increasing $\operatorname{Re}_{\tau}$, as it has almost vanished in the $\operatorname{Re}_{\tau} \approx 1400$ cases of Ref. [18]. This is further confirmation that small boxes with a single roll produce accurate statistics for the mean velocity profiles, and that a single-roll pair is enough.

In contrast, Fig. 6 shows the fluctuations of the streamwise velocity profile at the inner cylinder in wall units, i.e., $u^{\prime}=\left(\left\langle u^{2}\right\rangle_{\theta, z, t}-\langle u\rangle_{\theta, z, t}^{2}\right)^{1 / 2} / u_{\tau, i}$. The fluctuations show not only a dependence on $\lambda_{z}$, but also on the amount of rolls, increasing for the three-roll pair case when compared to the single-roll pair case with the same $\lambda_{z}$. These might be due to interactions between roll pairs and the different strength of each of the roll pairs. This is again consistent with the findings of Ref. [18], where the fluctuation statistics had not saturated to box-independent values for $\Gamma=4$. Some degree of collapse is seen near the inner cylinder $\left(r^{+}<60\right)$. However, the fluctuation peak of $u_{\theta}^{\prime}$ at $r^{+} \approx 12$ is substantially different across the considered cases, indicating that the fluctuations are both produced by the rolls itself and by roll-to-roll interactions.

To remove the effect of the large-scale structures on the fluctuations, we define the roll-less azimuthal velocity fluctuations as $u^{\star}=\left\langle\left\langle u^{2}\right\rangle_{\theta, t}-\langle u\rangle_{\theta, t}^{2}\right\rangle_{z}^{1 / 2} / u_{\tau, i}$ [7]. As detailed in Ref. [7], both definitions for the fluctuations give equivalent results for statistically homogeneous flows but give different results for flows which show some axial inhomogeneity. Figure 7 shows $u^{\star}$ at the inner cylinder for the four simulated cases. A similar behavior is seen across the three velocity components. 


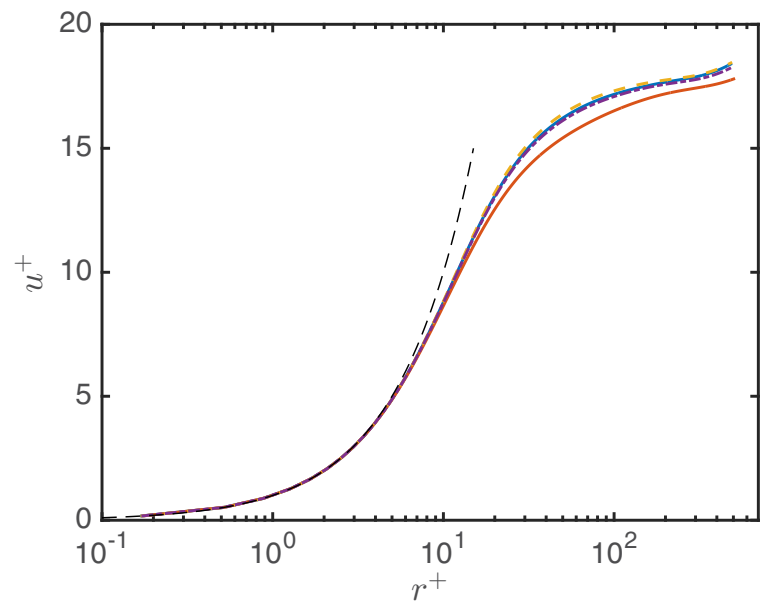

FIG. 5. Mean streamwise velocity profile at the inner cylinder in wall units for the four simulated cases. Symbols as in Table I. Thin black dashed line indicates $r^{+}=u^{+}$.

Near the inner cylinder $\left(r^{+}<60\right)$ (more markedly for $\left.u_{z}^{\star}\right)$ the profiles depend mainly on $\lambda_{z}$. The curve which shows the largest deviation from the others corresponds to the G7R2 case. However, for increasing $r^{+}$, i.e., as the bulk is approached, the collapse is better for the large $\Gamma$ cases, regardless of $\lambda_{z}$. From this it seems that the nature of the fluctuations captured by $u^{\star}$ changes in the bulk. Near the cylinder, it seems to capture the same sort of fluctuations as $u^{\prime}$, as this near-wall region is more axially homogeneous. Meanwhile, in the bulk it may be capturing fluctuations caused by shear and not by centrifugal forces. This is especially pronounced in the case of $u_{\theta}$ fluctuations. While in Fig. 6 some degree of collapse could be seen for the two cases with $\lambda_{z}=2.33$ and one- or three-roll pairs, we see that the collapse in $u_{\theta}^{\star}$ happens for cases with similar $\Gamma$ and different $\lambda_{z}$.

Finally, we show the premultiplied azimuthal and axial energy spectra of the azimuthal and axial velocity at midgap in Fig. 8. For the axial spectra, the low wave number part is dominated by the Taylor rolls. A clear maximum at the roll wave number $k_{T R}=2 \pi / \lambda_{z}$, followed by a dropoff for smaller wave numbers. The highest energy modes correspond to the harmonics of associated to the roll, i.e., $n k_{T R}$ with $n \in \mathbb{Z}$, and this results in a clear sawtooth pattern. The high wave number spectra collapse across all cases, consistent with the results of Ref. [18]. This leads to the $u^{\star}$ fluctuations for $\Gamma=7$. Remarkably, G2R1 and G7R3, i.e., the two cases with the same $\lambda_{z}$ and different roll number, do not collapse in this region. It appears that the details of the Taylor roll play a very small role in the fine features of the small scale fluctuations, and that a single-roll system cannot adequately
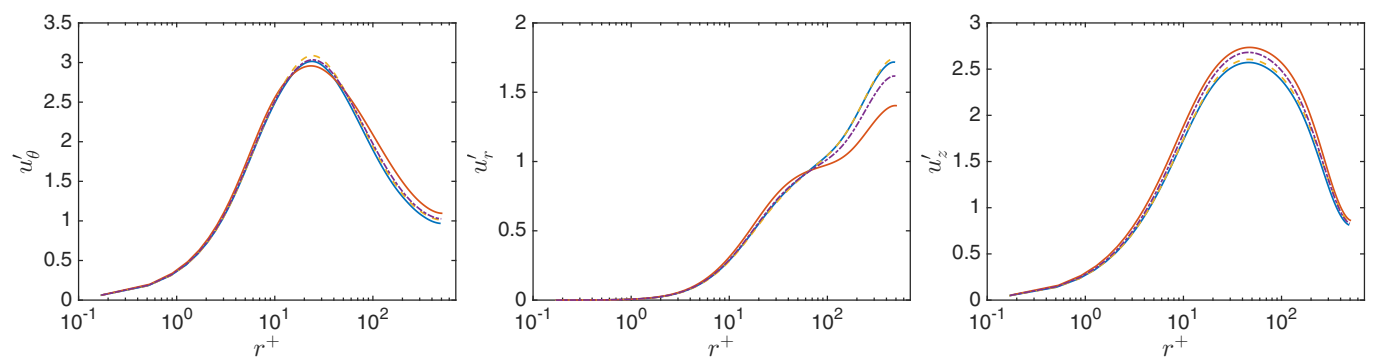

FIG. 6. Fluctuation velocity profiles at the inner cylinder in wall units for the four simulated cases. Symbols as in Table I. 

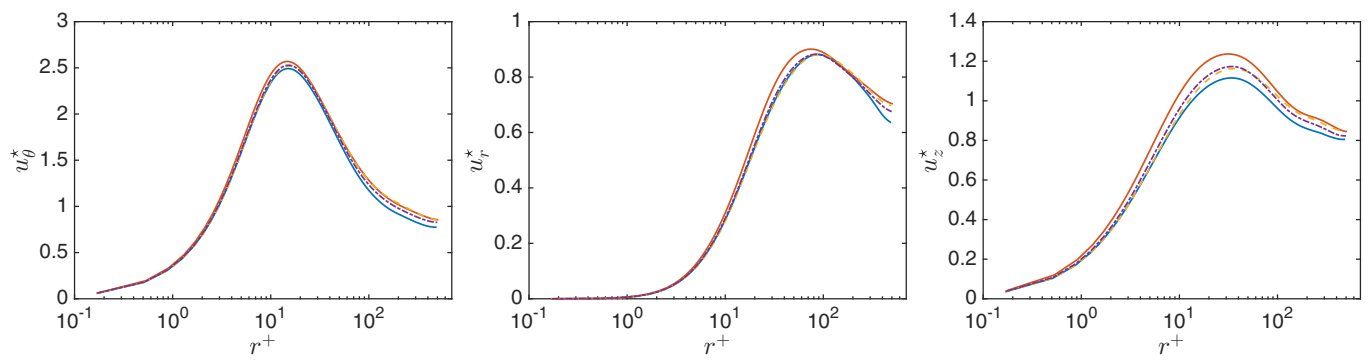

FIG. 7. Roll-less fluctuation velocity profiles at the inner cylinder in wall units for the four simulated cases. Symbols as in Table I.

capture the energy spectra of multiple rolls. This supports the conclusion that roll-to-roll interaction is important for determining the fluctuations, and especially the fluctuation peak at $r^{+}=12$.

The azimuthal spectra for both velocities are consistent with what was seen in Refs. [7,18]. The premultiplied spectra (i.e., multiplied by $k$ ) considered here have a clear maximum. We are thus simulating the largest energy-containing scales by using $n_{\text {sym }}=5$. However, there is a marked peak for the G7R2 case for wavelengths corresponding to one-quarter of the azimuthal extent of the domain, i.e., modes with a rotational symmetry of order 20 . This has not been reported before and might be due to a resonance of the box; a similar phenomena was seen in Ref. [18] for a computational
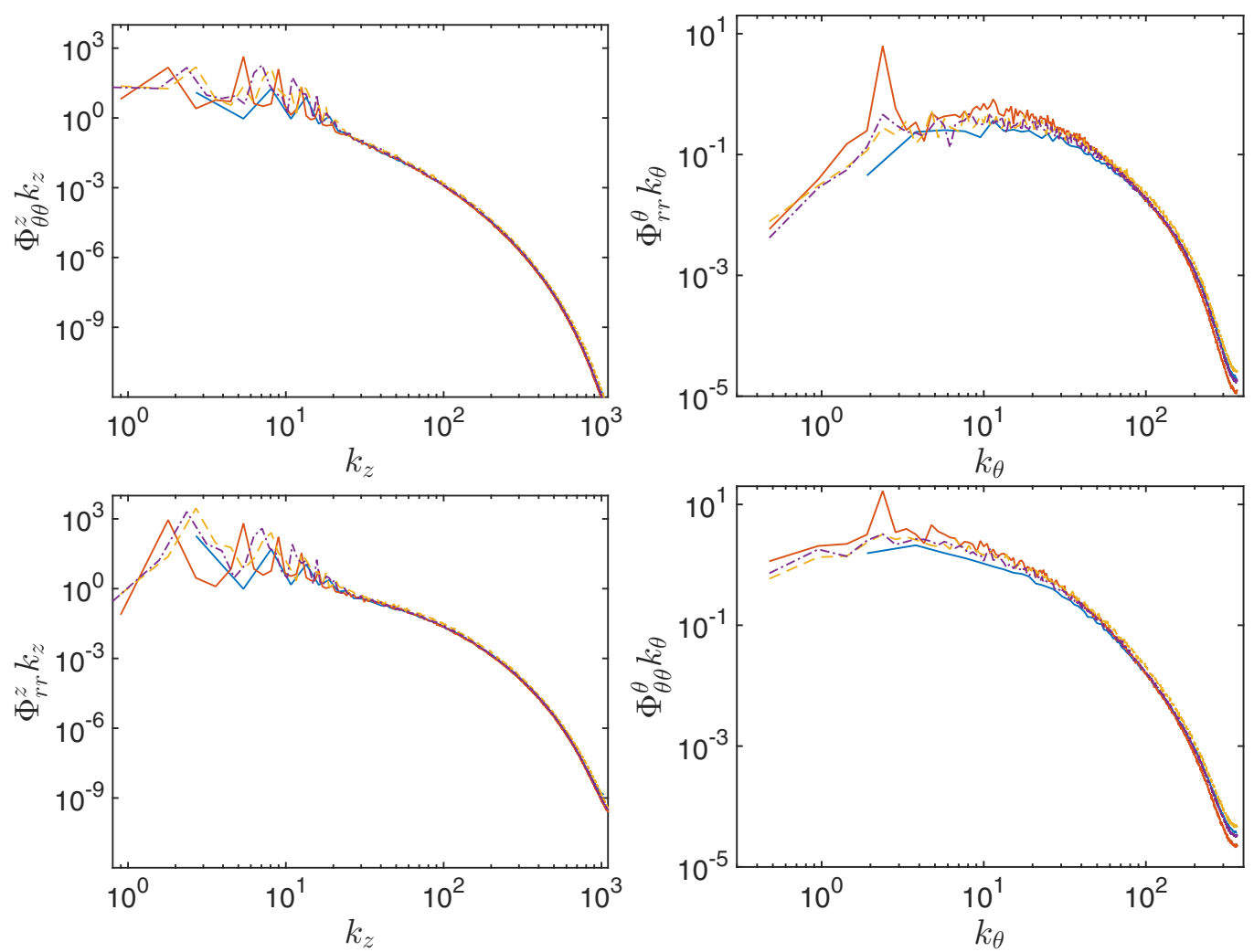

FIG. 8. Premultiplied velocity energy spectra $\Phi k$ in the axial (left) and azimuthal (right) directions for the azimuthal (left) and radial (right) velocities. Symbols as in Table I. 
box with $\Gamma=2.09$ and $n_{\text {sym }}=10$, which saw a very large increase of the velocity fluctuations at the midgap. These peaks could indicate certain "unnatural" resonances of the computational box, which exactly fit "wavy" azimuthal patterns.

\section{CONCLUSIONS}

In summary, we have run a series of simulations of TC flow using computational boxes with a large axial extent. The large-scale Taylor rolls were found to still be fixed, even for the largest axial aspect ratio of $\Gamma=8$. The rolls were found to preferably be in a "tall" configuration, i.e., to have a preferred wavelength of $\lambda_{z}>2$. Furthermore, two possible configurations were found to be stable for long periods of time for $\Gamma=7$, a two-roll pair configuration with $\lambda_{z}=3.5$ and a three-roll pair configuration with $\lambda_{z}=2.33$. A single-roll pair was found to give the same torque and mean velocity profiles as a simulation of three-roll pairs at with the same $\lambda_{z}$, consistent with the findings of Ref. [15]. A weak dependence on $\lambda_{z}$ was found for the torque, despite different fluctuation profiles in the bulk, supporting that the torque is dominated by the near-wall region. We have found that a small box with a single-roll pair is large enough to reproduce the torque and mean velocity profile turbulent TC flow, but it cannot reproduce the fluctuations and velocity spectra. This study in combination with Ref. [18] provides some promising evidence that at sufficiently high Reynolds number, the statistics of TC flow can reach box-size and roll-wavelength independence, provided the axial extent of the boxes is large enough. This will be confirmed only once these high Reynolds number simulations are actually conducted.

\section{ACKNOWLEDGMENTS}

We thank V. Spandan for extensive help in proofreading the paper. We acknowledge M. Bernardini, M. P. Encinar, J. Jiménez, P. Orlandi, S. Pirozzoli, Y. Yang, and X. Zhu for fruitful and stimulating discussions. We also gratefully acknowledge computational time for the simulations provided by SurfSARA on resource Cartesius through a NWO grant.

[1] M. A. Fardin, C. Perge, and N. Taberlet, The hydrogen atom of fluid dynamics: Introduction to the Taylor-Couette flow for soft matter scientists, Soft Matter 10, 3523 (2014).

[2] S. Grossmann, D. Lohse, and C. Sun, High-Reynolds number Taylor-Couette turbulence, Ann. Rev. Fluid Mech. 48, 53 (2016).

[3] R. Ostilla-Monico, E. P. van der Poel, R. Verzicco, S. Grossmann, and D. Lohse, Boundary layer dynamics at the transition between the classical and the ultimate regime of Taylor-Couette flow, Phys. Fluids 26, 015114 (2014).

[4] G. I. Taylor, Experiments on the motion of solid bodies in rotating fluids, Proc. R. Soc. Lond. A 104, 213 (1923).

[5] C. D. Andereck, S. S. Liu, and H. L. Swinney, Flow regimes in a circular Couette system with independently rotating cylinders, J. Fluid Mech. 164, 155 (1986).

[6] S. G. Huisman, R. C. A. van der Veen, C. Sun, and D. Lohse, Multiple states in highly turbulent TaylorCouette flow, Nat. Commun. 5, 3820 (2014).

[7] R. Ostilla-Mónico, R. Verzicco, S. Grossmann, and D. Lohse, The near-wall region of highly turbulent Taylor-Couette flow, J. Fluid Mech. 768, 95 (2016).

[8] R. Ostilla-Monico, E. P. van der Poel, R. Verzicco, S. Grossmann, and D. Lohse, Exploring the phase diagram of fully turbulent Taylor-Couette flow, J. Fluid Mech. 761, 1 (2014).

[9] D. Coles, Transition in circular couette flow, J. Fluid Mech. 21, 385 (1965).

[10] T. B. Benjamin and T. Mullin, Notes on the multiplicity of flows in the Taylor experiment, J. Fluid Mech. 121, 219 (1982). 
[11] D. P. Lathrop, Jay Fineberg, and H. L. Swinney, Transition to shear-driven turbulence in Couette-Taylor flow, Phys. Rev. A 46, 6390 (1992).

[12] B. Martinez-Arias, J. Peixinho, O. Crumeyrolle, and I. Mutabazi, Effect of the number of vortices on the torque scaling in Taylor-Couette flow, J. Fluid Mech. 748, 756 (2014).

[13] R. C. A. van der Veen, S. G. Huisman, On-Yu Dung, H. L. Tang, C. Sun, and D. Lohse, Exploring the phase space of multiple states in highly turbulent Taylor-Couette flow, Phys. Rev. Fluids 1, 024401 (2016).

[14] J. Jiménez, M. Bernardini, S. Pirozzoli, and P. Orlandi (private communication).

[15] H. Brauckmann and B. Eckhardt, Direct numerical simulations of local and global torque in Taylor-Couette flow up to Re $=30$ 000, J. Fluid Mech. 718, 398 (2013).

[16] H. J. Brauckmann and B. Eckhardt, Intermittent boundary layers and torque maxima in Taylor-Couette flow, Phys. Rev. E 87, 033004 (2013).

[17] H. Brauckmann, M. Salewski, and B. Eckhardt, Momentum transport in Taylor-Couette flow with vanishing curvature, J. Fluid Mech. 790, 419 (2015).

[18] R. Ostilla-Mónico, R. Verzicco, and D. Lohse, Effects of the computational domain size on DNS of Taylor-Couette turbulence with stationary outer cylinder, Phys. Fluids 27, 025110 (2015).

[19] A. Lozano-Durán and J. Jiménez, Effect of the computational domain on direct simulations of turbulent channels up to $\operatorname{Re}_{\tau}=4200$, Phys. Fluids 26, 011702 (2014).

[20] R. Verzicco and P. Orlandi, A finite-difference scheme for three-dimensional incompressible flow in cylindrical coordinates, J. Comput. Phys. 123, 402 (1996).

[21] E. P. van der Poel, R. Ostilla-Monico, J. Donners, and R. Verzicco, A pencil distributed finite difference code for strongly turbulent wall-bounded flows, Comp. Fluids 116, 10 (2015).

[22] V. Avsarkisov, S. Hoyas, M. Oberlack, and J. P. García-Galache, Turbulent plane Couette flow at moderately high Reynolds number, J. Fluid Mech. 751, R1 (2014).

[23] S. Pirozzoli, M. Bernardini, and P. Orlandi, Turbulence statistics in Couette flow at high Reynolds number, J. Fluid Mech. 758, 327 (2014).

[24] B. Dubrulle, O. Dauchot, F. Daviaud, P. Y. Longaretti, D. Richard, and J. P. Zahn, Stability and turbulent transport in Taylor-Couette flow from analysis of experimental data, Phys. Fluids 17, 095103 (2005).

[25] T. Tsukahara, H. Kawamura, and K. Shingai, DNS of turbulent Couette flow with emphasis on the large-scale structure in the core region, J. Turb. 7, 19 (2006).

[26] M. Bernardini, S. Pirozzoli, M. Quadrio, and P. Orlandi, Turbulent channel flow simulations in convecting reference frames, J. Comput. Phys. 232, 1 (2013).

[27] B. Eckhardt, S. Grossmann, and D. Lohse, Torque scaling in turbulent Taylor-Couette flow between independently rotating cylinders, J. Fluid Mech. 581, 221 (2007).

[28] P. Billant and F. Gallaire, Generalized Rayleigh criterion for non-axisymmetric centrifugal instabilities, J. Fluid Mech. 542, 365 (2005).

[29] J. Komminaho, A. Lundbladh, and A. V. Johansson, Very large structures in plane turbulent Couette flow, J. Fluid Mech 320, 259 (1996). 